\title{
The role of BAFF and G-CSF for rituximab-induced late-onset neutropenia (LON) in lymphomas
}

\author{
Daniel Tesfa ${ }^{1,3} \cdot$ Birgitta Sander $^{2} \cdot$ Henric Lindkvist ${ }^{1} \cdot$ Christer Nilsson $^{1} \cdot$ Eva Kimby $^{1} \oplus \cdot$ Hans Hägglund $^{1,4}$. \\ Björn E. Wahlin ${ }^{1} \cdot$ Monika Klimkowska $^{2}$ Jan Palmblad ${ }^{1}[0$
}

Received: 27 February 2021 / Accepted: 24 April 2021 / Published online: 18 May 2021

(c) The Author(s) 2021

\begin{abstract}
Mechanisms for late-onset neutropenia (LON) after rituximab treatment are poorly defined both for non-Hodgkin lymphoma (NHL) and for autoimmune disorders. We performed a case-control analysis of a prospective cohort of 169 evaluable consecutive rituximab-treated NHL patients to assess cytokines involved in neutro- and lymphopoiesis (G-CSF, SDF1, BAFF, APRIL) and inflammation (CRP) as possible LON mechanisms. Fifteen patients (9\%) developed LON (peripheral blood / $\mathrm{PB} /$ absolute neutrophil counts / $\mathrm{ANC} /<0.5 \mathrm{G} / \mathrm{L}$, all with marked depletion of $\mathrm{CD} 20^{+} \mathrm{B}$-lymphocytes in bone marrows); they were compared with 20 matched NHL controls without LON. At start of LON, significantly higher PB G-CSF and BAFF levels ( $P=0.0004$ and 0.006 , respectively), as well as CRP rises were noted compared to controls; these G-CSF and BAFF and most CRP values returned to levels of the controls in post-LON samples. G-CSF (but not BAFF) changes correlated to CRP rises (but not to ANC levels). BAFF levels correlated significantly to absolute monocyte counts and PB large granular lymphocyte counts (but not to ANC, C-CSF or CRP values). No changes of SDF1 or APRIL levels were noted. Neither LON cases nor controls displayed anti-neutrophil autoantibodies. Collectively, LON in NHL patients was timewise related to transient bursts of blood G-CSF and BAFF concentrations, suggesting that these neutro- and lymphopoiesis growth factors play a role in emergence of rituximab-induced LON, and that inflammation may be a trigger for G-CSF production during LON.
\end{abstract}

Keywords Late-onset neutropenia $\cdot$ Rituximab $\cdot$ G-CSF $\cdot$ BAFF $\cdot$ APRIL $\cdot$ SDF1

\section{Introduction}

Late-onset neutropenia (LON), following rituximab therapy for autoimmune diseases or lymphomas, is an unusual type of drug-induced neutropenia (NP), because it occurs between 1 and 12 months after completing rituximab therapy (and not during or shortly after intake as with most agranulocytosis-inducing drugs) [1-3]. The mechanisms for LON remain, however, poorly defined $[2,3]$.

Björn E. Wahlin

bjorn.wahlin@ki.se

Monika Klimkowska

monika.klimkowska@sll.se

1 The Hematology Center and Department of Medicine, Karolinska Institutet, Karolinska University Hospital Huddinge R51, 14186 Stockholm, Sweden

2 Department of Clinical Pathology and Cytology, Department of Laboratory Medicine, Karolinska University Hospital Huddinge, Karolinska Institutet, 14186 Stockholm, Sweden

3 Present Address: Swedish Orphan Biovitrum AB, 11276 Stockholm, Sweden

4 Division of Hematology, Department of Medical Sciences, Uppsala University, Uppsala, Sweden 
Recently, the role of BAFF (B-cell activating factor) has attracted attention in relation to rituximab treatment for rheumatic diseases [4-7]. Neutrophils and monocytes are important sources of BAFF and its release is induced by, for example, granulocyte colony-stimulating factor (G-CSF) [8-10]. BAFF, a member of the TNF family, plays a central role in the stimulation of B-lymphocytes and is a survival factor for transitional and mature B cells and immunoglobulin production [10]. High levels of BAFF may also induce autoantibodies and enhance the rheumatic process [5, 7].

After rituximab therapy, CD $20^{+}$B-lymphocytes are rapidly depleted and they reappear to the peripheral blood (PB) after 5-12 months [6, 11, 12], preceded by an elevation of peripheral blood (PB) levels of BAFF [4].Elevated BAFF levels have also been reported in LON in rheumatic diseases, with a return to basal levels after LON [6], yet its role in LON development is unclear, as well as the underlying mechanisms involved in BAFF elevation. For instance, concomitant infection/ inflammation involving transient G-CSF production may promote BAFF release, as shown in other settings [8-10]. In addition, we and others reported that LON in lymphoma and rheumatic patients is related to the possession of certain $B A F F$ and FCGR3 gene polymorphisms $[13,14]$, suggesting possible genedrug interactions.

To assess if there is a relationship between emergence of LON in NHL patients and coexistent perturbations of BAFF production, we performed a case-control analysis of a prospective cohort of 174 consecutive NHL patients treated with rituximab. We used $\mathrm{PB}$ and bone marrow (BM) samples obtained at the time of detection of LON and in post-LON samples. We also analyzed G-CSF (a BAFFpromoting cytokine $[8,10]$, also central for emergency neutropoiesis $[9,15])$ and C-reactive protein (CRP, as a sign of inflammation/infection). Further, we studied APRIL (A Proliferation-Inducing Ligand, also secreted by myeloid and other cells, being a proliferation and maturation factor for B-lymphocytes $[6,9,16]$, as well as stromal-derived factor-1 (SDF-1/CXCL12), a molecule involved in neutrophil egress from and return to the BM $[11,17]$. Finally, we analyzed the presence of anti-neutrophil autoantibodies and large granular lymphocytes, LGL (as consequences of autoimmunity-promoting BAFF rises [5, 7] and being central for development of autoimmune NPs), shifts of T-lymphocyte subpopulations and NK cell numbers in PB and BM. Thus, LON was used as a model to analyze possible mechanisms for a drug-induced neutropenia (DINP).

\section{Patients and methods}

\section{Study design and patients}

\section{Inclusions}

The study, approved by the Ethics committee at Karolinska Institutet, Stockholm, Sweden, was performed in accordance with the Helsinki declaration. All patients gave their written informed consent before initiation of rituximab treatment.

We included prospectively 174 consecutive adult NHL patients, treated with rituximab at the Hematology Center, Karolinska University Hospital, Huddinge, Stockholm, Sweden. Figure 1 summarizes the design and essentials of the study.

\section{Patients}

Patients were treated according to standard care protocol at the discretion of the treating physician; however, all received rituximab. Median age was 62 years (range 26-83). Ninety were males and 79 females. Seventeen patients were treated with rituximab alone, at $375 \mathrm{mg} / \mathrm{m}^{2}$. The others received rituximab, at the same dose, in combination with chemotherapy; details are given in Table 1. CBCs (complete

174 NHL patients were entered 1 mo after rituximab therapy (103 DLBCL, $31 \mathrm{FL}, 14 \mathrm{MCL}, 12 \mathrm{CLL}, 5 \mathrm{MbW}, 9 \mathrm{MZL})$

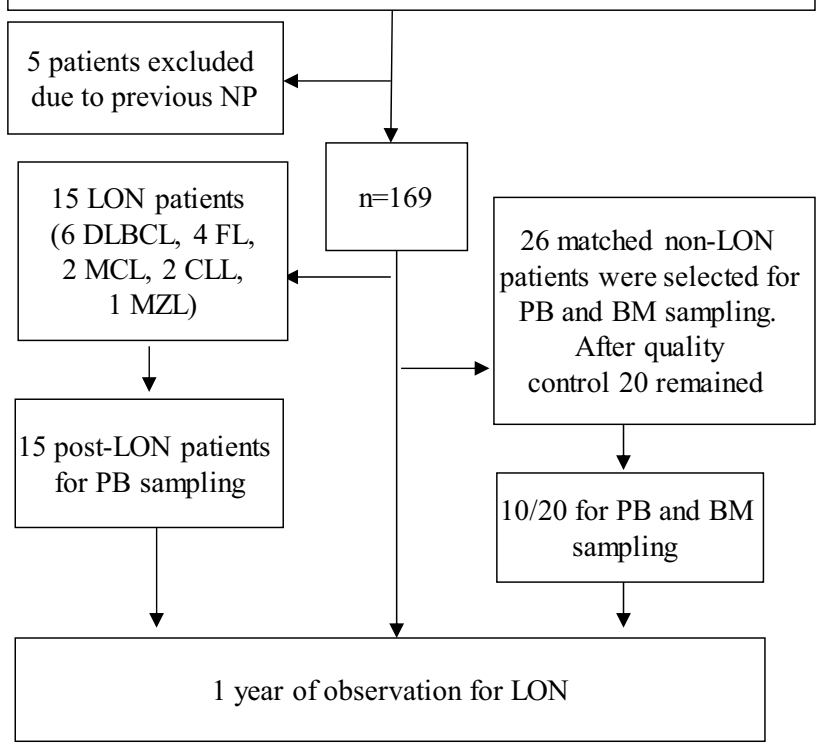

Fig. 1 Consort flow diagram of the study. $L O N$ late onset neutropenia, $N P$ neutropenia, $D L B C L$ diffuse large B-cell lymphoma, $F L$ follicular lymphoma, $M C L$ mantle cell lymphoma, $C L L$ chronic lymphocytic leukemia, $M b W \mathrm{Mb}$ Waldenström (macroglobulinemia), $M Z L$ marginal zone lymphoma 
Table 1 Demographic characteristics and treatments of the LON and non-LON NHL control patients

\begin{tabular}{llll}
\hline & $\begin{array}{l}\text { LON-patients } \\
n=15\end{array}$ & $\begin{array}{l}\text { Controls } \\
n=26\end{array}$ & $P$-value \\
\hline Age, years, median \pm SD (min-max) & $58 \pm 11.8(35-74)$ & $61.5 \pm 15,9(27-86)$ & 0.6 \\
Sex, $n(\%)$ & & & \\
Males & $8(53 \%)$ & $14(54 \%)$ & 0.8 \\
Females & $7(47 \%)$ & $12(46 \%)$ & 0.8 \\
Ann Arbor stage at diagnosis, $n(\%)$ & & & 0.8 \\
I-II & $2(13 \%)$ & $6(23 \%)$ & 0.8 \\
III-IV & $13(87 \%)$ & $20(77 \%)$ & 0.8 \\
Treatment type and intensity, $n(\%)$ & & & 0.8 \\
De novo treatment & $8(57 \%)$ & $16(62 \%)$ & \\
Previous rituximab therapy & $6(43 \%)$ & $9(35 \%)$ & 0.8 \\
Rituximab maintenance therapy & $1(7 \%)$ & $2(8 \%) 1.0$ & 0.6 \\
Relapse treatment & $6(43 \%)$ & $10(38 \%)$ & 0.9 \\
Antracycline based treatment & $8(57 \%)$ & $4(69 \%)$ & 0.7 \\
Fludarabine based treatment & $2(14 \%)$ & $8(31 \%)$ & 0.6 \\
HSCT treatment & $5(36 \%)$ & $4(15 \%)$ & 0.18 \\
Other treatment modalities & $4(29 \%)$ & $5300 \pm 953.9$ & 0.15 \\
Methotrexate (HDMTX) dose, mg, mean \pm SD & $5200 \pm 848.5$ & $4253.9 \pm 738.8$ & \\
Rituximab dose, mg, mean \pm SD & $3850 \pm 598.4$ & &
\end{tabular}

$S D$ standard deviation; IQR interquartile range; HSCT autologous hematological stem cell transplantation; HDMTX high-dose methotrexate blood counts) and PB C-reactive protein (CRP) levels were obtained routinely every fortnight to month.

\section{Exclusions}

Medical records were reviewed for the time of two years before the start of rituximab therapy and all patients with a previous history of NP were excluded. Hence, 169 patients were included for LON analysis (Fig. 1 and Supplementary Material).

\section{Definition of LON}

LON is defined as an otherwise unexplained $\mathrm{PB}$ absolute neutrophil count (ANC) of $\leq 0.5 \mathrm{G} / \mathrm{L}$ (corresponding to grade 4 neutropenia according to National Cancer Institute Common Toxicity Criteria (NCI-CTC) [18], and agranulocytosis as $\mathrm{ANC} \leq 0.1 \mathrm{G} / \mathrm{L}$, starting earliest four weeks after the termination of rituximab therapy. Absolute PB monocyte counts (AMC) were considered normal if $0.2-0.8 \mathrm{G} / \mathrm{L}$. All patients were followed for, at least, 12 months after rituximab treatment [19] (Supplementary Material).

\section{Controls}

A control group within the same cohort of NHL patients was established simultaneously with detection of a LON episode. This comparison was done to control for confounding factors for the NP. See Supplementary Material for details. In the end, 26 control subjects were included (Table 1). Full sets of PB samples were obtained from 20 of these controls. In addition, a subgroup $(n=10)$ of these 20 patients volunteered to also provide BM samples.

\section{Peripheral blood (PB) and bone marrow (BM) evaluations}

In LON patients, PB samples were obtained twice: first, at detection of LON ("LON samples") and, second, after resolution of LON ("post-LON samples"). BM samples were obtained at the first PB sampling. We also retrieved clinical PB ANC and AMC immediately preceding onset of a LON episode (i.e.1/-2/ weeks). In patients given G-CSF because of LON, BM and PB samples were collected before start of G-CSF treatment (except for one case) and, at least, one week after stopping that therapy (except for two cases); all data shown are for those without ongoing or recent G-CSF treatment. LON and post-LON PB samples were also used for serology and serum cytokine tests and CRP (analyzed according to hospital routines). See also Supplementary Material.

Relative LGL cell abundance was analyzed by light microscopy on routinely stained PB smears, as advised by Loughran and Lamy [20].

In the matched controls, BM $(n=10)$ and PB samples $(n=20)$ were also collected and processed at time points 
corresponding to the detection of LON in the LON patients, to control for potential time-related effects for BM regeneration after the last rituximab infusion and chemotherapy.

BM flow cytometry was performed by fluorescence immunophenotyping for $\mathrm{B}$ and $\mathrm{T}$ lymphocytes, and for natural killer (NK) cells, as described [14, 19]. For analysis of B-cells, fluorochrome-conjugated monoclonal antibodies to B-cells antigens CD19 and CD20 were used. For analysis of T-cells and NK-cells, anti-CD3, anti-CD4, anti-CD8, anti-CD16 and anti-CD56 antibodies were used. See also Supplementary Material.

B cell depletion (analyzed by flow cytometry) was analyzed as percentage of BM cells expressing CD20.

\section{Enzyme linked immunosorbent assay (ELISA)}

Serum levels of human BAFF, APRIL, G-CSF and plasma levels of SDF-1 (a k a CXCL12) were analyzed using Quantikine immunoassays (R\&D Systems Europe) according to the manufacturer's instructions.

\section{Anti-neutrophil antibodies}

The test panel included granulocyte agglutination test (GAT) and granulocyte immunofluorescence tests (GIFT), followed by monoclonal antibody-specific immobilization of granulocyte antigen (MAIGA) test [21]. See Supplementary Material for details.

\section{Statistical analyses}

Values are presented as mean \pm SD or median (and interquartile range, IQR) depending on value distributions. The chi-squared test and Fisher's exact test were used to compare patient characteristics, treatments and proportions. Wilcoxon's matched pairs/signed rank test was performed to assess differences in lymphocyte subpopulations at different times for paired samples of LON and non-LON-matched control patients. Associations between time to LON/duration of LON, ANC/AMC, cytokine, CRP levels and ANC were analysed by Spearman's correlation analysis. All tests were 2-sided and $\mathrm{P}$ values less than 0.05 were considered significant.

\section{Results}

\section{LON characteristics}

Fifteen patients (8.8\% of all patients in the cohort) presented with LON (eight males and seven females, median age 58 years; Fig. 1). LON patients and their 26 matched non-LON controls had similar demographics, NHL subdiagnoses and treatments (including rituximab and methotrexate) as the other NHL patients (Table 1 and Supplementary Table 1).

After initial lymphoma treatment, all LON and control patients had recovered normal ANCs, were in clinical remission and had no other identifiable causes of NP than LON. Post-LON PB samples were obtained from all 15 LON patients (still being in remission) within 3 weeks after LON resolution. In the end, blood samples from 20 non-LON controls (out of the initially selected 26) remained because of inappropriate collection and assaying.

The characteristics of LON patients are given in detail in Supplementary material. Briefly, the median time to onset of LON was 96 days and median duration of LON was 17 days (Figs. 2A, B). Four patients were febrile at LON detection and had moderate CRP rises $(<59$, reference value $<4 \mathrm{mg} / \mathrm{L}$ ); in addition, three more had minor CRP rises $(<20)$. Nine patients received short term treatment with G-CSF (Fig. 2A, B).

The ANC. The median nadir ANC was $0.2 \mathrm{G} / \mathrm{L}$; thus, all LON patients developed severe NP (Fig. 3A). None of the 20 non-LON controls with available CBC, corresponding to the time to $\mathrm{LON}$ of their matched pairs, displayed ANC $<1.5 \mathrm{G} / \mathrm{L}(P<0.0001 ;$ Fig. $3 \mathrm{~A})$.

The $A M C$ for $\mathrm{LON}$ patients were similar to those of non-LON controls $(P>0.05$ for all comparisons; Fig. 3B) and did not correlate to change of ANCs $(P>0.05)$; thus, those with the most pronounced ANC drop did not raise their AMC more than those with the least pronounced ANC drops.

Time to LON and LON duration correlated significantly, in that those with a short time to LON had longer duration of LON than those with a long time to LON $(P=0.048$; Fig. 2C). LON-patients with a previous HSCT ( $n=4 ; 27 \%$ of all LON patients) had similar incidences and complications to LON as those without (Fig. 2A, B).

Post-LON data. At time for post-LON sampling, all LON patients had regained normal ANCs, AMCs remained unchanged, raised CRP values had returned to normal in 3/5 and remained moderately elevated in $2 / 5$.

B-lymphocyte depletion at start of LON. Only LONpatients displayed a complete depletion of $\mathrm{BM} \mathrm{CD} 20^{+}$cells (Fig. 4), whereas LON-patients and non-LON controls did not differ in numbers of $\mathrm{CD} 19^{+} \mathrm{CD} 20^{-} \mathrm{B}$-lymphocytes in the $\mathrm{BM}$, as analysed by flow cytometry.

BM lymphocyte subpopulations, myeloid, NK and LGL cells and relation to LON. The numbers of $\mathrm{CD}^{+}$(T-cells) and $\mathrm{CD}^{-}{ }^{-} \mathrm{CD} 56^{+}$cells (NK cells) as well as numbers of $\mathrm{CD}^{+}{ }^{+}$or $\mathrm{CD} 8^{+}$cells or in $\mathrm{CD} 4^{+} / \mathrm{CD} 8^{+}$ratio (i.e. subpopulations of T-cells) did not differ significantly between the LON or control groups (Fig. 4). However, we did observe 


\section{Time to LON, days}
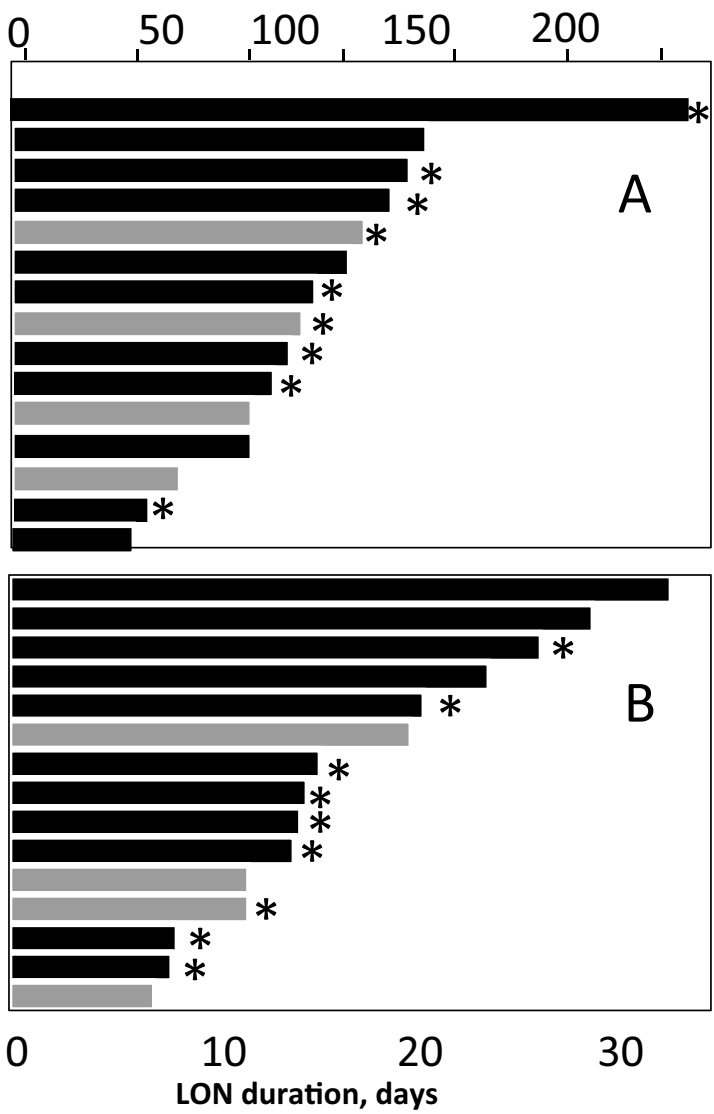

Fig. 2 Time to LON (panel A), duration of LON (panel B), correlation between time to and duration of LON (panel $\mathbf{C}$ ) and correlation between PB levels of G-CSF and duration of LON (panel D). Grey

significantly higher numbers of $\mathrm{CD}^{+} \mathrm{CD}^{+} 6^{+}$(T/NK-cells) in LON than in control patients (Fig. 4).

Percentages of $\mathrm{CD} 33^{+}$and $\mathrm{CD} 117^{+}$cells were significantly higher in LON patients at start of LON than in controls (Fig. 4).

No significant associations were noted for relations of $\mathrm{CD}^{+} 56^{+}$cells to ANC and $\mathrm{AMC}$ (data not shown).

$L G L$ cells were found in $\mathrm{PB}$ samples in LON patients trendwise more often than in controls (14.3\% of all lymphocytes vs $9.2 \%$, respectively; $P=0.057)$. The percentages of LON patients did not correlate to numbers of $\mathrm{CD}^{+} \mathrm{CD} 56^{+}$ (T/NK-cells; $P>0.05$ ).

\section{Serum BAFF levels}

Previously, we showed that B-lymphocyte depletion coincided timewise with LON, and regenerated B-lymphocyte numbers with normalized ANC; this suggested that LON may be related to factors for proliferation of B-lymphocytes, such as BAFF [6, 14, 17, 19].
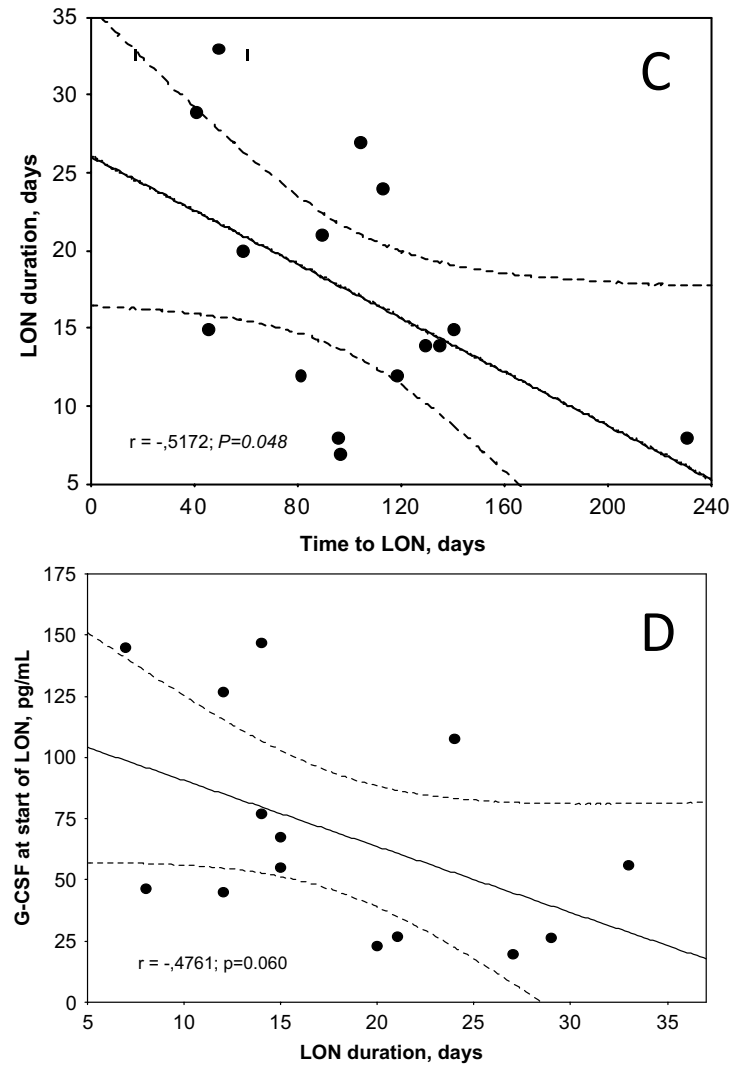

bars in panel A represent patients with a previous HSCT. Asterisks denote patients treated with filgrastim/i.v. antibiotics during LON

At onset of LON, serum BAFF values were significantly higher in LON patients compared to non-LON controls $(p=0.018)$. In post-LON samples, BAFF levels decreased ( $p=0.0002$ for the difference between LON and post-LON samples), and were then significantly lower than in nonLON controls ( $p=0.002)$ (Fig. 3C).

Considering that BAFF is produced to a large extent by monocytes and neutrophils $[8-10,15]$ and that the LON patients were severely neutropenic, but not monocytopenic at LON onset, we asked if the AMC correlated with BAFF levels at onset of LON and we found a significant correlation ( $r=0.639, P=0.025$; Fig. 5A). There were no significant correlations between BAFF levels at onset of LON and the $\mathrm{ANC}, \mathrm{CD} 33^{+}$or $\mathrm{CD} 117^{+}$cells at this time, neither was there a significant correlation of BAFF changes and the ANCs over the LON period (data not shown). Furthermore, BAFF levels did not correlate with CRP levels at start of LON or with time to or duration of LON $(P>0.05$; data not shown). However, BAFF figures correlated positively significantly to numbers of $\mathrm{CD} 3{ }^{+} \mathrm{CD} 56^{+}$ 

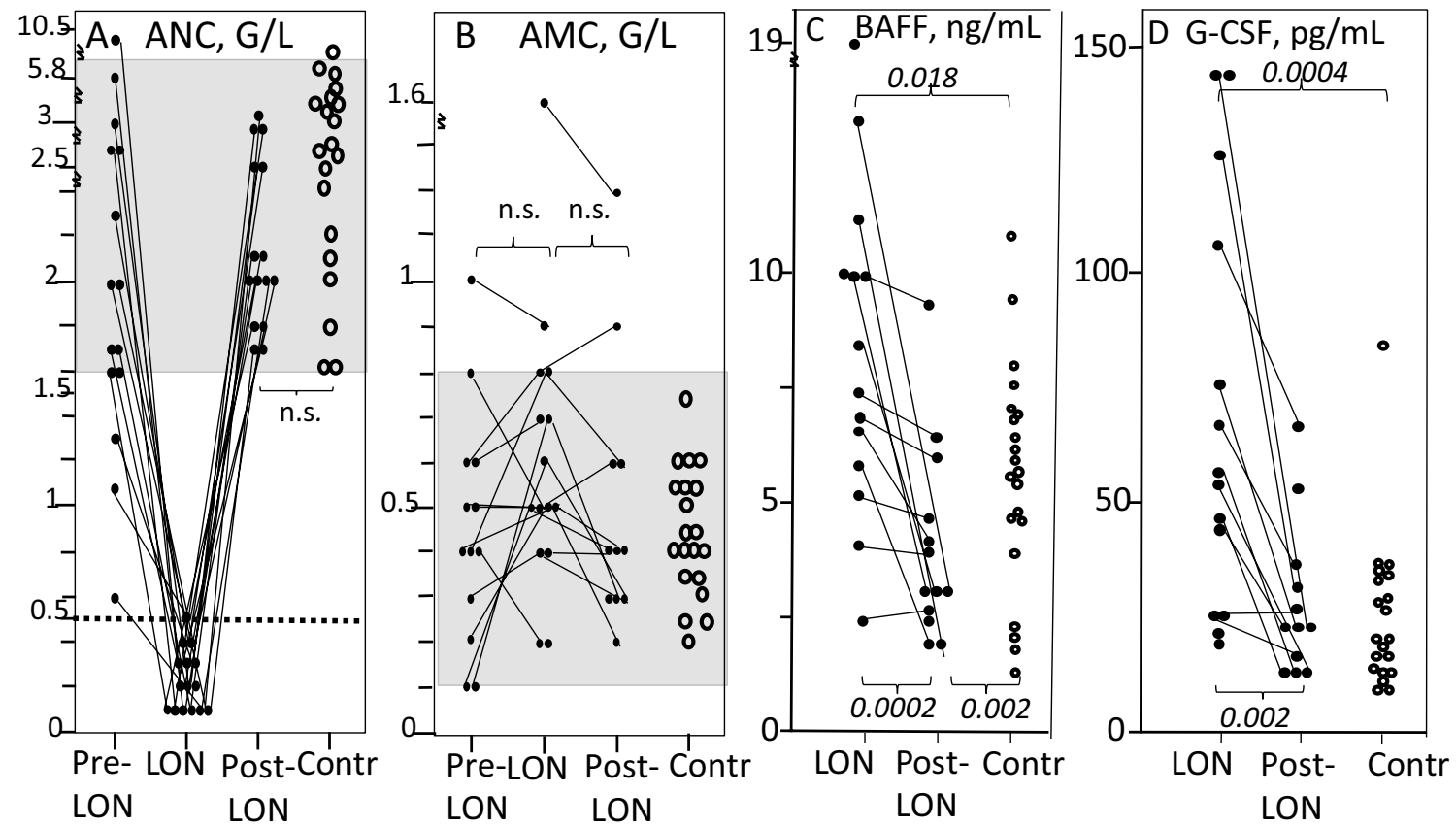

Fig. 3 Peripheral blood (PB) absolute neutrophil counts (ANC, panel A), and absolute monocyte counts (AMC, panel B). Panel $\mathbf{C}$ shows PB BAFF levels and panel D PB G-CSF values. Grey areas represent reference values for healthy controls. $P$-values are given close to

brackets. n.s. no significant differences. Solid circles $=\mathrm{LON}$ patients, open circles $=$ matched non-LON patients. Pre-LON samples are those obtained immediately prior to the start of $\mathrm{LON}, \mathrm{LON}=$ at diagnosis of LON, and post-LON = upon recovery from LON

Fig. 4 Flow cytometry analysis of BM cells. Results are given as percent of cells in the mononuclear gate, except for the CD4/CD8 ratio. $P$-values are given between the boxes. Mean (filled squares), SE (boxes) and $1.96 *$ SE (whiskers) values. Statistical significance $(p<0.05)$ between LON and controls is indicated by italic figures
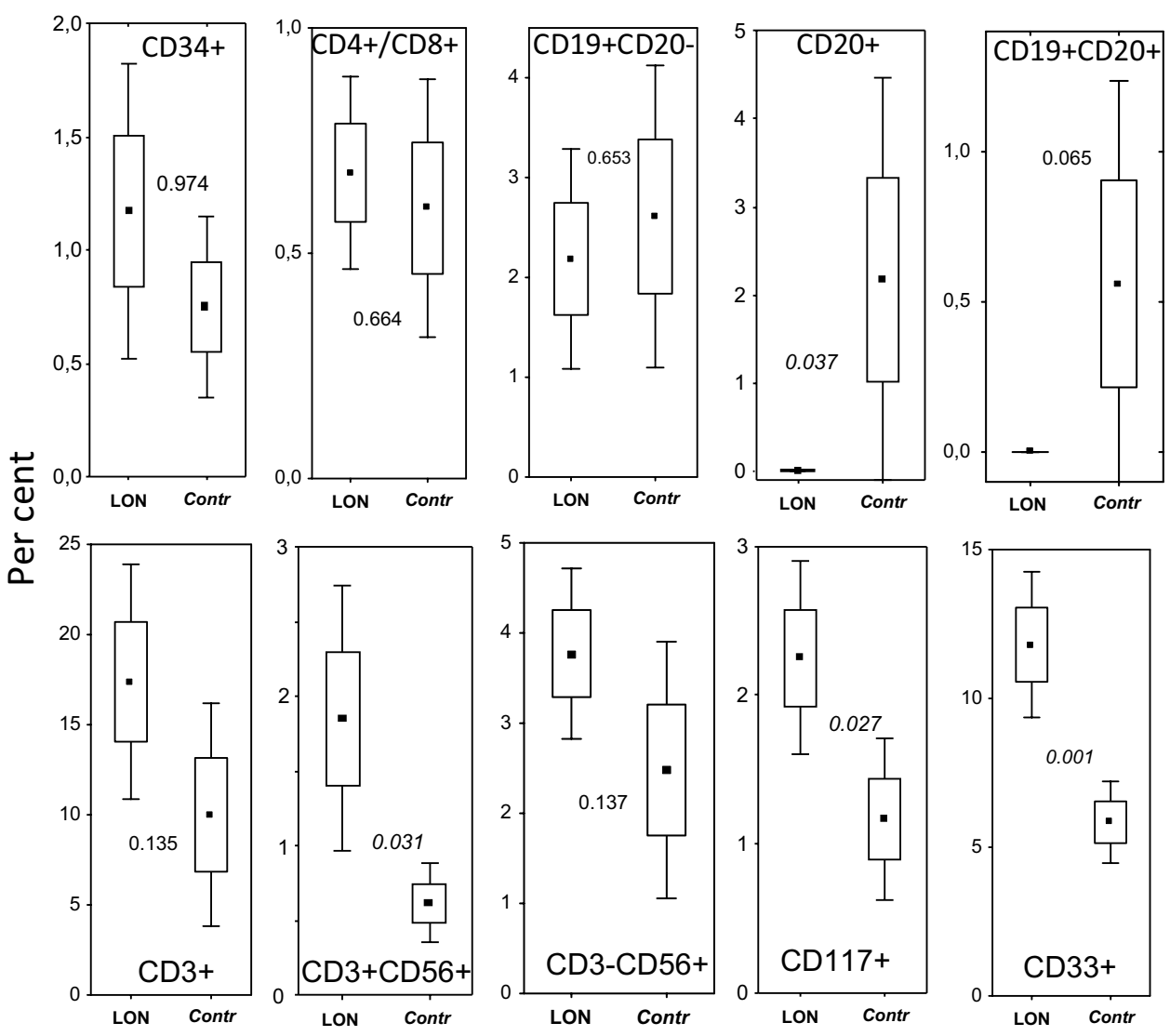

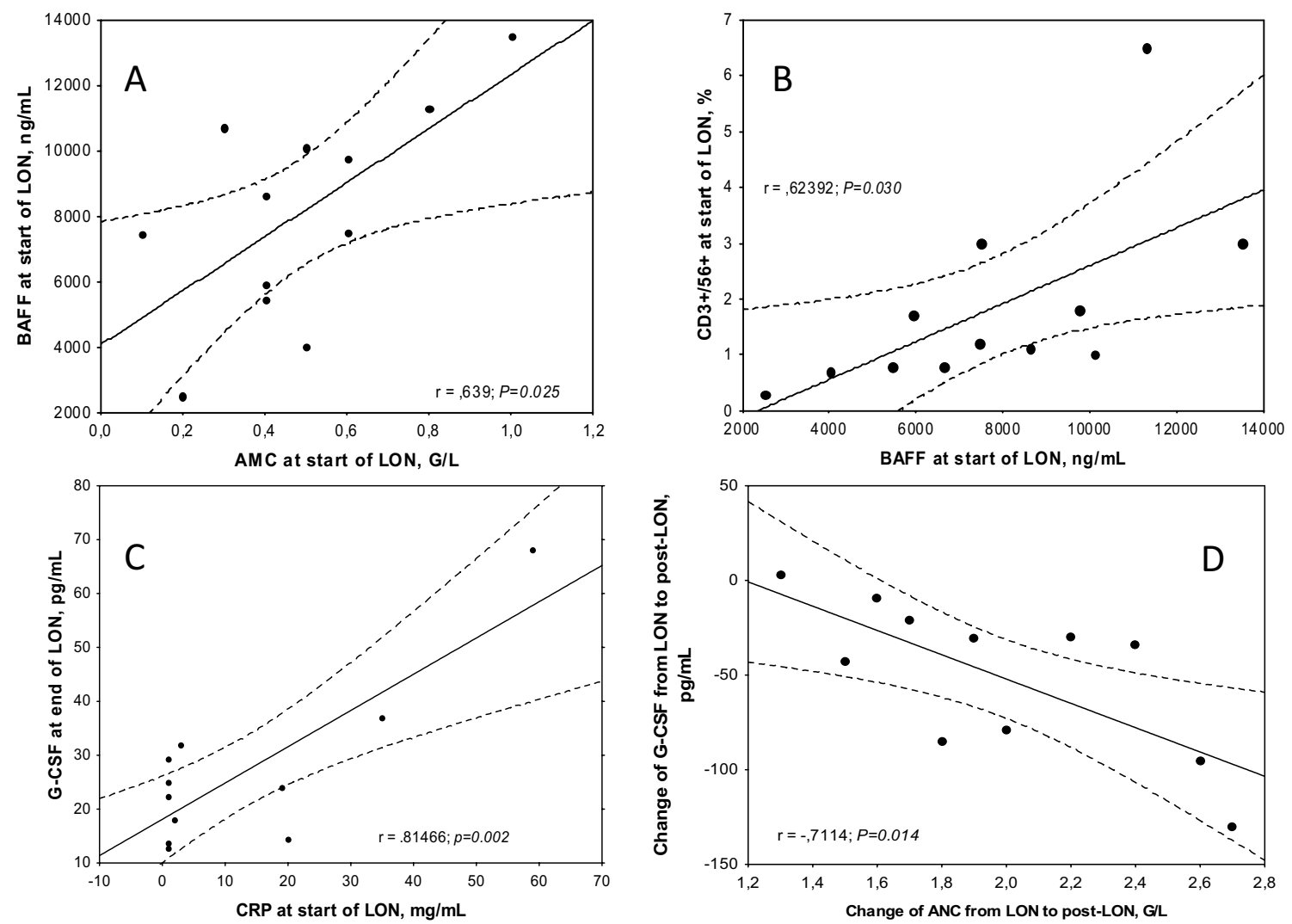

Fig. 5 Spearman's correlations between: Panel A peripheral blood absolute monocyte counts (AMC) and BAFF concentrations, both at start of LON; Panel B BAFF concentrations and CD3 + CD56 + cells in the bone marrow, both at start of LON; Panel $\mathbf{C}$ concentrations of CRP at start of LON and G-CSF at end of LON; and Panel $\mathbf{D}$ change

of absolute neutrophil counts (ANC) and G-CSF concentrations, both as the change between start of LON and post-LON values. $P$-values are given after the $r$-values. Significand correlations are marked in italics

(T/NK-cells; $P=0.030 ;$ Fig. 5B). Thus, positive relations of BAFF levels to PB monocyte and $\mathrm{CD}^{+} \mathrm{CD}^{+} 6^{+}$(T/NKcells) counts were found, but not to signs of inflammation (CRP) or of NP (i.e. ANC).

\section{Serum APRIL levels}

In contrast to BAFF, APRIL values did not change over LON time (Supplementary Table 2). There were no significant correlations between APRIL levels, on one hand, and, on the other, BAFF, CRP or AMC.

\section{Serum G-CSF levels}

G-CSF values were significantly higher at start of LON than in post-LON samples $(p=0.002)$ and when compared with non-LON controls $(p=0.0004)$. Post-LON values did not differ from those of non-LON controls (Fig. 3D). G-CSF values at $\mathrm{LON}$ start showed a trend towards a negative correlation to duration of LON, i.e. those with the highest G-CSF

values at start of LON tended to have the shortest LON duration (Fig. 2D).

Considering data indicating a relationship between $\mathrm{PB}$ levels of G-CSF and infection/inflammation in chronic autoimmune NP individuals (but not to grade of NP) [22], we asked if similar relations might be found in the acute agranulocytosis of this study. There was a trendwise significant positive correlation $(p=0.053)$ between the PB levels of CRP and G-CSF at LON start, and a highly significant correlation between CRP at LON start and the G-CSF levels at end of LON ( $p=0.002$; Fig. 5C). Moreover, raises of ANC from nadir to post-LON correlated significantly to reductions of G-CSF levels over the LON period ( $p=0.014$, Fig. 5D). Thus, during the LON period significant interactions of NP, inflammation and G-CSF levels were detected.

Next, since G-CSF is reported to be a strong inducer of BAFF secretion [8-10, 15], we looked for relations of G-CSF and BAFF at start, end of and changes over LON period, but found no significant ones $(P>0.05$, data not shown). Likewise, no correlation was found for G-CSF 
levels and $\mathrm{CD}_{3} 3^{+}$or $\mathrm{CD} 117^{+}$cells at LON start (data not shown). Thus, the role of G-CSF as inducer of BAFF secretion in an acute NP such as LON remains elusive, but may depend on different kinetics of changes.

\section{Plasma SDF-1 values}

PB SDF1 values did not differ significantly during the LON period (Supplementary Table 2); thus, there was no indication of a systemic effect on release (or re-entry) of neutrophils over the blood-BM barrier.

\section{Anti-neutrophil antibodies and LGL cells}

Since BAFF rises have been associated with emergence of autoimmune phenomena [7] we analysed if LON associated BAFF rises might trigger development of autoantibodies to neutrophils, but none of the LON patients or the matched non-LON patients displayed GAT, GIFT or MAIGA positivity. Then, we asked if emergence of LGL-cells, previously considered to be part of the autoimmune spectrum of NPs $[23,24]$, related to BAFF rises at start of LON. A significant positive correlation ( $P=0.012$; Supplementary Fig. 1$)$ was found. Thus, BAFF may play a role for the appearance of LGL-cells in LON.

\section{Discussion}

LON is a unique form of drug-induced neutropenia (DINP) since it may start 1-12 months after last administration of the drug, whereas other DINPs start during or up to one week after treatment [25]. The reported incidence of rituximab-induced LON (5-27\%) is also manifold higher than for traditional DINPs, $(0.2-2 \%$ of treated patients) [26, 27]. LON represents a unique opportunity to analyze mechanisms for one form of DINP.

Our major findings of LON characteristics agree with previous descriptions $[1-7,19]$. Three novel observation were that (1) the longer time to LON presentation, the shorter was LON duration, that (2) BAFF and G-CSF increases was found at LON onset, and (3) LON duration was shorter in those with the highest G-CSF levels. The reason for these phenomena is not known but may be related to genetic factors $[13,14]$ and feedback regulatory systems.

One unresolved question is if all rituximab-treated patients develop LON, but that those cases with a mild clinical course escape detection, implying that LON is an integral part of (side) effects of rituximab and not an idiosyncratic reaction. Our major argument against this hypothesis is that no NP cases were identified among the well-matched controls. However, to prove or reject the hypothesis of LON being an integral part of rituximab treatment requires even more frequent PB samplings than we obtained. Thus, we can, but not unequivocally so, argue that LON appears to be an idiosyncratic reaction.

The mechanisms for LON still remain elusive. Here, we focused primarily on cytokines that might be parts of the process, alone or in consort.

One previous study suggested that perturbation of SDF-1/ CXCL12 during B-lymphocyte recovery retards neutrophil egress from the BM [11] and thus causes NP. We did not find any significant differences in PB SDF-1 levels here, making such an explanation less likely. Moreover, the paucity of BM mature neutrophils in LON speaks against such effect [28].

A role for NK cells in NP has been discussed [29] and may be a mechanism for LON. However, we found no changes of NK cell counts (but of $\mathrm{CD} 3+56+$ cells, i.e. T/ NK cells). This issue needs phenotyping of NK cells to be resolved. Interestingly, BAFF levels at LON start correlated with frequencies of these $\mathrm{CD} 3+56+$ cells (Fig. 5B). The somewhat higher PB LGL counts in our LON patients remain elusive for the LON process but may be part of a BAFF-related mechanism also involving Fas/Fas ligand. Our finding agrees with other studies [23, 24].

A suggested deficiency of the major growth factor for neutrophils, G-CSF [15, 30], was not found here; instead, we noted rises at LON onset and reductions to levels of controls in post-LON samples (Fig. 3D). Moreover, several statistically significant correlations were found between G-CSF changes, on the one hand, and of CRP and of ANC, on the other. Based on this and data from G-CSF variations during cyclic NP and in autoimmune NP [22], we suggest that G-CSF rises observed here can be reactions to concomitant neutropenic infections as well as feed-back reactions to the abruptly emerging agranulocytosis. Since these phenomena are highly interrelated, experimental systems are needed for dissecting the chain of events. However, we cannot rule out a temporary absolute and/or functional deficiency of the cognate receptor for G-CSF, CSF3R, due to paucity of neutrophil precursors in the BM [28] that might reduce cellular uptake of G-CSF and thus accumulation of G-CSF in the PB.

Since BAFF has been pointed out as a possible mechanism for LON [4, 6, 17], we evaluated this hypothesis. Some findings here support a role of BAFF in the LON process. The first is the high BP BAFF levels at LON onset and subsequent reductions within 2-3 weeks, when ANCs are normalized. A second reason is that high G-CSF levels might suppress BAFF production at later stages of LON, which might be reflected here in the lower BAFF levels (than in controls) in post-LON samples [30]. However, there are confounding observations. One is that we did not find statistical correlations of BAFF levels to the degree of NP (i.e. ANCs), G-CSF or inflammation (i.e. CRP) levels. Another is that BAFF levels peaked at time of the lowest 
ANC, and lowest BAFF levels occurred at resolution of LON. These findings apparently contradict PB neutrophils as major BAFF producers in $\mathrm{LON}[8,9]$. In addition, $\mathrm{LON}$ BMs show a maturation arrest at the (pro)myelocyte stage of granulopoiesis [28], indicating not only PB but also BM neutrophil reductions.

The positive correlation of BAFF levels to PB AMC suggests a role for monocyte series cells as producers of BAFF, as suggested [7-9]. Despite no significant change in the AMC during LON, PB AMCs may not reflect the total body monocyte/macrophage pool size. Our data may suggest a relation between the acute NP, monocytic cells and regulation of BAFF generation. Although we did not note a significant correlation between changes of BAFF and G-CSF, other studies are needed to find if BAFF rises are secondary to emergency-induced G-CSF secretion [9].

The reason for the discordant reactions of BAFF and APRIL during LON remains to be revealed but has been observed previously $[4,6]$.

The propensity of high BAFF production to enhance autoimmune processes $[5,7]$ made us look at presence of anti-neutrophil antibodies. Such antibodies were previously suggested to be a mechanism for LON [1], but none of the patients here had detectable levels.

A weakness of our study is the rather few LON cases. However, LON is a rare side-effect and our prospective material is among the larger published so far. Another weakness is that statistical correlations for data over time may be negative because of different kinetics of changes.

One strength is that we have prospectively matched controls (for diagnoses etc.) for comparisons at similar time points as when LON developed. Another is $\mathrm{PB}$ and $\mathrm{BM}$ sampling within days after start of LON and that the same persons were followed over time.

In conclusion, our current investigations provide evidence for a complex interaction of myelo- and lymphopoiesis and suggest that a burst of BAFF PB levels may be associated with the development of LON although it is hard to distinguish between a causal and a correlative relationship. Moreover, we confirm in the LON subjects that inflammation and the acute NP is driving G-CSF rises. However, there is a need for larger patient materials as well as experimental studies to validate this model of a DINP.

Supplementary Information The online version contains supplementary material available at https://doi.org/10.1007/s12032-021-01516-8.

Acknowledgements We thank Inger Vedin, Ph.D., was instrumental for the cytokine analyses, and Professor Bengt Fadeel, Karolinska Institutet, Stockholm, Sweden, for help with the study planning.

Funding Open access funding provided by Karolinska Institute. The study was supported by grants from the Karolinska Institutet Ph.D. study fund (KID) and Stockholm County Council (ALF project).
Data availability Yes.

\section{Declarations}

Conflict of interest DT is currently employed by Swedish Orphan Biovitrum AB, SE-112 76 Stockholm, Sweden. At the time of the study: no other conflicts of interest have been declared. All other authors: no conflict of interest are reported.

Ethical approval This study was approved of the Ethics Committee of the Karolinska Institutet.

Informed consent All patients gave written consent.

Open Access This article is licensed under a Creative Commons Attribution 4.0 International License, which permits use, sharing, adaptation, distribution and reproduction in any medium or format, as long as you give appropriate credit to the original author(s) and the source, provide a link to the Creative Commons licence, and indicate if changes were made. The images or other third party material in this article are included in the article's Creative Commons licence, unless indicated otherwise in a credit line to the material. If material is not included in the article's Creative Commons licence and your intended use is not permitted by statutory regulation or exceeds the permitted use, you will need to obtain permission directly from the copyright holder. To view a copy of this licence, visit http://creativecommons.org/licenses/by/4.0/.

\section{References}

1. Voog E, Morschhauser F, Solal-Celigny P. Neutropenia in patients treated with rituximab. N Engl J Med. 2003;348:2691-4.

2. Tesfa D, Palmblad J. Late-onset neutropenia following rituximab treatment: incidence, clinical features and possible mechanisms. Expert Rev Hematol. 2011;4:619-25.

3. Wolach O, Shpilberg O, Lahav M. Neutropenia after rituximab treatment: new insights on a late complication. Curr Opin Hematol. 2012;19:32-8.

4. Vallerskog T, Heimbürger M, Gunnarsson I, et al. Differential effects on BAFF and APRIL levels in rituximab-treated patients with systemic lupus erythematosus and rheumatoid arthritis. Arthritis Res Ther. 2006;8:R167.

5. Ehrenstein MR, Wing C. The BAFFling effects of rituximab in lupus: danger ahead? Nat Rev Rheumatol. 2016;12:367-72.

6. Parodis I, Söder F, Faustini F, et al. Rituximab-mediated late-onset neutropenia in systemic lupus erythematosus-distinct roles of BAFF and APRIL. Lupus. 2018;27:1470-8.

7. Shabgah AG, Shariati-Sarabi Z, Tavakkol-Afshari J, Mohammadi $\mathrm{M}$. The role of BAFF and APRIL in rheumatoid arthritis. J Cell Physiol. 2019;234:17050-63.

8. Scapini P, Bazzoni F, Cassatella MA. Regulation of B-cell-activating factor (BAFF)/B lymphocyte stimulator (BLyS) expression in human neutrophils. Immunol Lett. 2008;116:1-6.

9. Parsa R, Lund H, Georgoudaki AM, et al. BAFF-secreting neutrophils drive plasma cell responses during emergency granulopoiesis. J Exp Med. 2016;213:1537-53.

10. Smulski CR, Eibel H. BAFF and BAFF-receptor in B cell selection and survival. Front Immunol. 2018;9:2285 (eCollection 2018).

11. Dunleavy K, Hakim F, Kim HK, et al. B-cell recovery following rituximab-based therapy is associated with perturbations in 
stromal derived factor-1 and granulocyte homeostasis. Blood. 2005; 106:795-802.

12. Leandro MJ, Cambridge G, Ehrenstein MR, Edwards JC. Reconstitution of peripheral blood B cells after depletion with rituximab in patients with rheumatoid arthritis. Arthritis Rheum. 2006;54:613-20.

13. Li SC, Chen YC, Evens AM, et al. Rituximab-induced late-onset neutropenia B-cell lymphoma correlates with $\mathrm{Fc}$ receptor Fc $\gamma$ RIIIa 158(V/F) polymorphism. Am J Hematol. 2010;85:810-2.

14. Ajeganova $\mathrm{S}$, Tesfa $\mathrm{D}$, Hägglund $\mathrm{H}$, et al. Effect of FCGR polymorphism on the occurrence of late-onset neutropenia and flarefree survival in rheumatic patients treated with rituximab. Arthritis Res Ther. 2017;19:44.

15. Boettcher S, Manz MG. Regulation of inflammation- and infection-driven hematopoiesis. Trends Immunol. 2017;38:345-57.

16. Baert L, Manfroi B, Casez O, Sturm N, Huard B. The role of APRIL - a proliferation inducing ligand-in autoimmune diseases and expectations from its targeting. J Autoimmun. 2018;95:179-90.

17. Terrier B, Ittah M, Tourneur L, et al. Late-onset neutropenia following rituximab results from a hematopoietic lineage competition due to an excessive BAFF-induced B-cell recovery. Haematologica. 2007;92:e20-3.

18. Cancer Therapy Evaluation Program. Common toxicity criteria version 20 (CTC). Bethesda: National Institutes of Health, National Cancer Institute; 1999.

19. Tesfa $\mathrm{D}$, Ajeganova $\mathrm{S}$, Hägglund $\mathrm{H}$, et al. Late-onset neutropenia following rituximab therapy in rheumatic diseases: association with B lymphocyte depletion and infections. Arthritis Rheum. 2011;63:2209-14.

20. Lamy T, Moignet A, Loughran TP Jr. LGL leukemia: from pathogenesis to treatment. Blood. 2017;129:1082-94.

21. Palmblad J, Nilsson CC, Höglund P, Papadaki HA. How we diagnose and treat neutropenia in adults. Expert Rev Hematol. 2016;9:479-87.

22. Bux J, Hofmann C, Welte K. Serum G-CSF levels are not increased in patients with antibody-induced neutropenia unless they are suffering from infectious diseases. Br J Haematol. 1999;105:616-7.

23. Papadaki T, Stamatopoulos K, Stavroyianni N, et al. Evidence for T-large granular lymphocyte-mediated neutropenia in rituximab-treated lymphoma patients: report of two cases. Leuk Res. 2002;26:597-600.

24. Stamatopoulos K, Papadaki T, Pontikoglou C, et al. Lymphocyte subpopulation imbalances, bone marrow hematopoiesis and histopathology in rituximab-treated lymphoma patients with late-onset neutropenia. Leukemia. 2008;22:1446-9.

25. Andrès E, Zimmer J, Mecili M, Weitten T, Alt M, Maloisel F. Clinical presentation and management of drug-induced agranulocytosis. Expert Rev Hematol. 2011;4:143-51.

26. Andersohn F, Konzen C, Garbe E. Systematic review: agranulocytosis induced by nonchemotherapy drugs. Ann Intern Med. 2007; 146:657-65.

27. Tricta F, Uetrecht J, Galanello R, et al. Deferiprone-induced agranulocytosis: 20 years of clinical observations. Am J Hematol. 2016;91:1026-31

28. Tesfa D, Gelius T, Sander B, et al. Late-onset neutropenia associated with rituximab therapy: evidence for a maturation arrest at the (pro)myelocyte stage of granulopoiesis. Med Oncol. 2008;25:374-9.

29. Jaeger BN, Donadieu J, Cognet C, et al. Neutrophil depletion impairs natural killer cell maturation, function, and homeostasis. J Exp Med. 2012;209:565-80.

30. Day RB, Bhattacharya D, Nagasawa T, Link DC. Granulocyte colony-stimulating factor reprograms bone marrow stromal cells to actively suppress B lymphopoiesis in mice. Blood. 2015;125:3114-7.

Publisher's Note Springer Nature remains neutral with regard to jurisdictional claims in published maps and institutional affiliations. 